\title{
An Overview of Wavelets for Image Processing for Wireless Applications
}

\author{
Dom Osborne ${ }^{a, c}$, Derek Rogers ${ }^{b}$, Jaganath Mazumdar $^{a, d}$, Reginald Coutts ${ }^{c}$ and Derek \\ Abbott $^{a, c}$ \\ ${ }^{a}$ Centre for Biomedical Engineering (CBME), The University of Adelaide, SA 5005, Australia \\ ${ }^{b}$ Motorola Australia Pty Ltd, 2 Second Ave Mawson Lakes, Adelaide, SA 5095, Australia \\ ${ }^{c}$ Department of Electrical and Electronic Engineering, The University of Adelaide, SA 5005, \\ Australia \\ ${ }^{d}$ Department of Applied Mathematics, The University of Adelaide SA, 5005, Australia
}

\begin{abstract}
The implementation of wavelets in differing areas of signal processing has been a popular research area over the last decade. However, utilising this technology in compressing two dimensional signals, such as digital images is relatively new. Wavelet compression has many distinct advantages over earlier compression methods, the most important of which is suitability to error protection as well as the ability to precisely truncate the compressed bitstream to achieve a desired bit rate for transmission. In this paper some of the recently emerging technologies pertaining to wavelet coding of images will be reviewed, particularly with the use of wireless channels. These developments include techniques to filter images that have been degraded through the addition of noise as well as reconstructing parts of images that have been lost as a result of the fading that characterises wireless mobile environments.
\end{abstract}

Keywords: Wavelet transform, wireless channels, channel coding, edge reconstruction, de-noising.

\section{INTRODUCTION}

One of the most rapidly expanding technological areas in communications is telematics. This can be defined as the use of computers or information systems to receive, store and distribute information over a distance implementing the use of a mobile telecommunications system. The communication of images and video over wireless environments in particular is one of the most popular subsets of telematics. This has applications in a large field of areas including mobile handset communication, videoconferencing, telerobotics and telemedicine. For wide acceptance of wireless image communication, fast transmission, low error rates, scalability for different receiving devices and high subjective image quality is mandatory. One of the first steps in improving the effectiveness of this communication is to facilitate the best possible type of image compression. JPEG (Joint Picture Experts Group) established the first international standard for continuous tone, colour and monochrome image compression in 1992. This technology involved using encoders and decoders that were DCT (Discrete Cosine Transform) based, which is a type of two dimensional fourier transform. Unfortunately, this type of transform results in images containing blocking artifacts - especially obvious at low bit rates, which is at present an unavoidable constraint if the communication methodology is mobile and wireless. In recent years wavelet based transform techniques, such as JPEG2000 ${ }^{1}$ have become a cutting edge technology in signal processing and a favorable alternative to the DCT for image compression. Section 2.3 discusses the main improvements in utilising this type of coding for wireless communication. Some work has been reviewed in Sections 3.1 to 3.3 that has involved expanding on applying wavelet image technologies for wireless communication.

\section{IMPLEMENTING WAVELET CODING FOR IMAGES}

\subsection{What are Wavelets?}

Similar to sinusoids used in Fourier analysis, wavelets are used as a type of basis function to represent arbitrary functions. There are many different sorts of wavelets and the type used can vary with the application. Individual wavelet functions are characterised by definition over a finite interval and a mean value of zero. Figure 1 is 


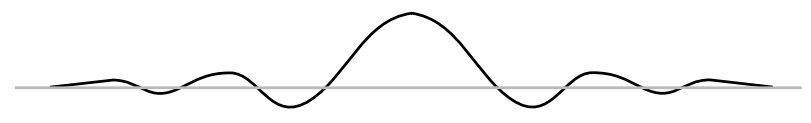

Figure 1: Time Representation of a generalised Wavelet

a generalised diagram of a wavelet. There are many types of wavelets, which can be smooth or compactly supported. Wavelets may be based on mathematical or just simple filter expressions. Once the type of wavelet (or mother wavelet) $\psi(t)$ is established, it is possible to make translations and dilations to analyse signals in frequency and time respectively. Graphically, this can be achieved by using short, high frequency wavelets to obtain detailed time information and limited frequency information or by using long, low frequency wavelets to obtain large frequency information and limited time information. This type of frequency-time trade off ${ }^{2}$ is illustrated in Figure 2. Many classes of functions can be represented by wavelets in a more compact way.

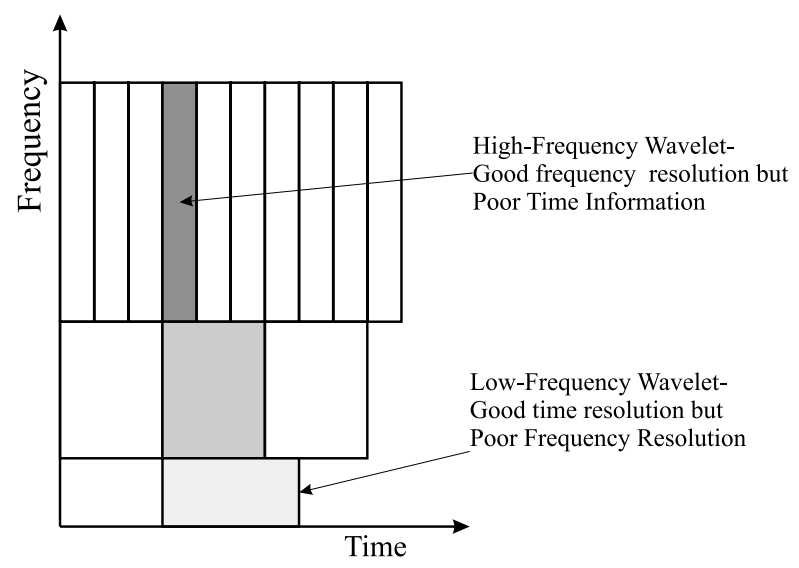

Figure 2: Time vs Frequency for Wavelet Transforms

This could include functions with discontinuities or sharp spikes that would typically take fewer wavelet basis functions than sinusoids to obtain a comparable approximation. According to the paper by Mallatt and Hwang, referenced at, ${ }^{3}$ this makes wavelets useful in data compression.

\subsection{The Discrete Wavelet Transform (DWT)}

For the purposes of digital signal processing (DSP), wavelets are represented by a series of samples as in Figure 3. When a wavelet transform is applied, the wavelet sequence is convolved across the signal sequence being

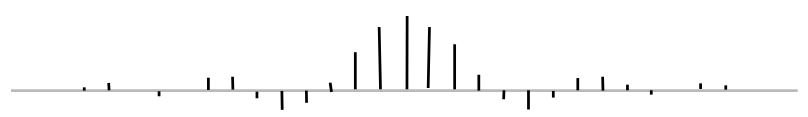

Figure 3: Discrete time representation of a generalised wavelet

transformed. Analogous to the fourier transform, this is the means of obtaining wavelet coefficients. ${ }^{4}$ From a mathematical perspective, discrete-time wavelets have the general form:

$$
\psi^{(a, b)}(t)=\frac{1}{\sqrt{a}} \psi\left(\frac{t-b}{a}\right)
$$

The variables $a$ and $b$ are used to scale and expand the wavelet, $\psi$. $a$ is used to scale the wavelet (by powers of two) width and $b$ is used to translate the wavelet in integer amounts. To analyse data at different resolutions a 
scaling function $w(t)$ is used in conjunction with the base (or mother) wavelet:

$$
W(t)=\sum_{k=-1}^{N-2}(-1)^{k} C_{k} \psi(2 t+k)
$$

In equation, $C_{k}$ are the wavelet coefficients which satisfy the constraints

$$
\sum_{k=0}^{N-1} C_{k}=2 \quad \text { and } \sum_{k=0}^{N-1} C_{k} C_{b}=2 \delta_{b, 0}
$$

In this case, $\delta$ is the delta function and $b$ is the location index. This allows for the defining coefficients to be varied according to the wavelet system used. ${ }^{5}$

\subsection{Comparing DCT and Wavelet-Based Image Coding}

According to a paper by Xiong, Ramchandran, Orchard and Zhang, ${ }^{6}$ for a fair comparison between DCT and wavelet-based image coding it is important to weigh the complexity and performance. It is well established that the hardware and software implementations of the DCT is much simpler than that using the wavelet transform. The simplest algorithm for the two-dimensional $8 \times 8$ DCT involves only 54 multiplications, ${ }^{7}$ however calculating the discrete wavelet transform depends on the length of the wavelet filters, involving at least one multiplication per coefficient. This would be an important factor to consider if mobile hand held devices with limited battery capacity are used to encode or decode the images by computing the inverse wavelet transform. Despite an obvious increase in system complexity, wavelet compression offers two main advantages over the DCT. These include:

- Improved Scalability - This is because the wavelet transform process can be repeated for as many iterations as needed. As the decoder operates inversely to the encoder, decoding can cease if entire resolution of the original image is not required. The decoder used performs the inverse process, whereby higher frequency information is superimposed on lower frequency image data. The decoder can stop any time if needed, as full resolution of the original image may not be required This would again be dependent on the resolution of the display device being used.

- Higher efficiency at low bit rates - Artifacts generated by removing high spacial frequency information are not as easily noticed as those generated using the DCT, ${ }^{1}$ where level differences frequently occur between adjacent $8 \times 8$ DCT blocks as causing abrupt changes in image intensity. As there are much more smooth transitions between coded blocks, low bit rate wavelet coded images are less objectionable to the viewer. This means that fewer basis functions are required for comparable image quality utilising the DCT. The few remaining wavelet coefficients can be quantised and organised implementing a procedure called Embedded Zerotree Wavelet encoding (EZW), developed by J. Shapiro in $1993,{ }^{8}$ which compactly codes these coefficients.

\subsection{The Tree Structure and Subband Coding}

When implemented on images, a wavelet transform transforms data from the space domain to the joint spacescale domain. Consequently in order to compress the transformed image it is necessary to code the coefficient values as well as their position in space. As a result of the transform, it is convenient to represent images using a tree structure because of the subsampling that takes place during the transform. This process is referred to as subband coding and takes place by dilating and translating a specific wavelet such that it has the effect of a filter. This takes place in the following way:

1. The image is filtered horizontally by convolving it with the high pass filter, that extracts high spatial frequency information and high detail.

2. The image is separately convolved horizontally with a complementary low-pass filter, which removes high frequency information leaving low frequencies. 
3. This results in two sub-images that contain high or low horizontal frequency information.

4. These two sub-images can then be convolved with each of the filters separately, to obtain four sub-images. This process is illustrated in Figure 4.

5. This process is repeated for the sub-image block containing low horizontal and low vertical frequencies (LL) to obtain higher band decomposition filter trees. From an image perspective, this process is also conveyed in Figure 5. ${ }^{5,9}$

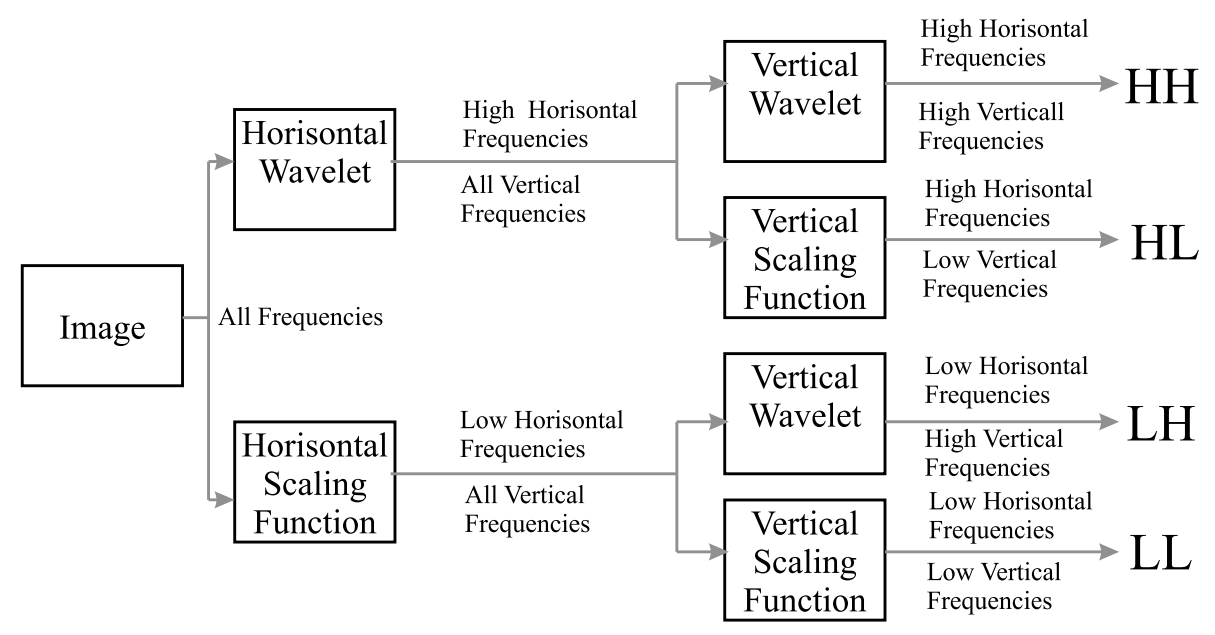

Figure 4: Dividing image into four sub-images

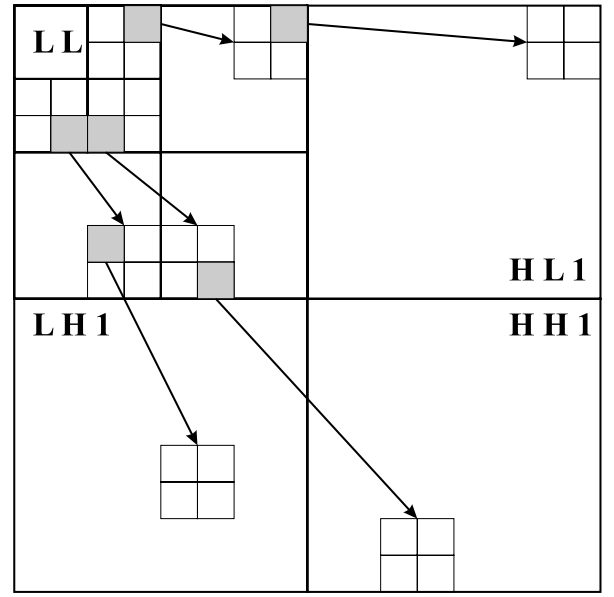

Figure 5: Relations between wavelet coefficients in different subbands

For the case of JPEG2000, the new international wavelet-based still image coding standard, the wavelet transform process is applied on $8 \times 8$ tiles. $^{10}$ This transformation can be visualised in Figure 6 . The resultant wavelet coefficients are quantised and can then be source encoded using arithmetic coding after implementing embedded zerotree scanning. This process facilitates the natural coding of the wavelet coefficient values as well as their position in space. 


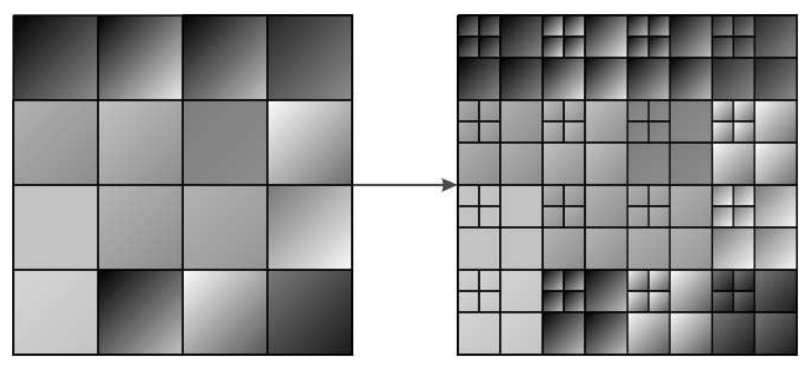

Figure 6: Division of Image Components and 2-D DWT

\section{IMAGE DENOISING AND RECONSTRUCTION TECHNIQUES USING WAVELETS}

\subsection{Image Denoising}

Despite being more suitable to low bit rate environments, such as mobile channels, the addition of noise to wavelet-based images can still present itself as a problem. As a result, it is desirable to remove the noise if possible.

At the most basic level, additive noise to an image can effectively be suppressed by thresholding the wavelet coefficients. The principle behind this is that small wavelet coefficients are most likely to be due to noise, where as large coefficients typically represent important signal features. ${ }^{11}$ Some more recent wavelet based denoising techniques make use of the image context ${ }^{12}$ as well as the spatial correlations between image wavelet coefficients. ${ }^{13}$ Denoising based on the context of the image is discussed in Section 3.1.1.

\subsubsection{Image Denoising based on Image Context}

This idea was presented by Chang et al, ${ }^{12}$ and is based on the important tradeoff between keeping key signal features and removing noisy wavelet coefficients. This technique involves choosing adaptive thresholds so that it can be possible to distinguish between coefficients that are mainly due to the signal and those due to noise. This is unlike the approach presented by Donoho et al, ${ }^{11}$ that involved a consistent threshold value. Because continuous tone images (such as photographs) have changing characteristics, the wavelet decomposition makes it possible to differentiate these areas. High energy areas typically represent image features containing sharp variations such as edges and textures, resulting in large wavelet coefficients. Low energy areas represent image features that correspond to smooth regions, typically containing small wavelet coefficients. This can be verified in the wavelet decomposition of Barbara 7, where white pixels represent large wavelet coefficients, and black signifies smooth regions containing small coefficients. When noise is added, it has the effect of increasing the average value of the wavelet coefficients. Smooth areas in particular would contain wavelet coefficients dominated by noise. As a consequence, it would be desirable to remove most of these coefficients. However in regions where there are edges and textures, wavelet coefficients have more energy due to the signal than as a result of additive noise, which may not be visible in these areas anyway. As a result it is convenient to keep these coefficients to ensure that important image information is retained. Chang et al ${ }^{12}$ distinguished between the low and high energy regions and changed the wavelet coefficients using a spatially adaptive thresholding technique. This involved modelling each wavelet coefficient as a realisation from a given probability distribution. This typically took the form of a generalised Gaussian distribution.

\subsection{Edge Reconstruction for Low Bit-Rate Wavelet-Compressed Images}

As discussed in Section 2.3, wavelet-based image compression is advantageous over the earlier block-based compression techniques. However the bandwidth constraints of mobile environments involve coding images at very low bit rates. This facilitates the requirement to discard much of the image detail resulting in obvious artifacts that are most visible around sharp edges and discontinuities. These are referred to as ringing effects and blurring effects. Distortion around these edges is perceptually objectionable and cannot be easily avoided if images are required to be transmitted at low bit rates. ${ }^{14}$ This type of edge distortion is easily seen in the eyelashes of Lena in Figures 8 and 9. In order to improve the image quality in these circumstances, post processing is a useful 


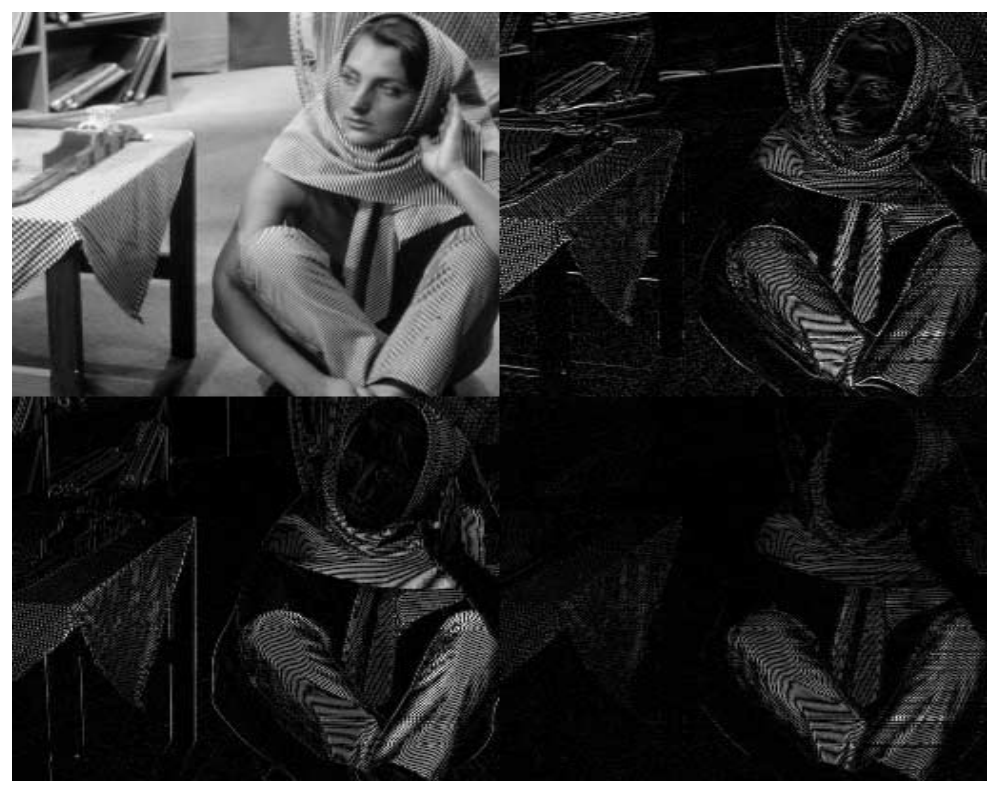

Figure 7: One level wavelet decomposition of Barbara. (Figure generated in Matlab)

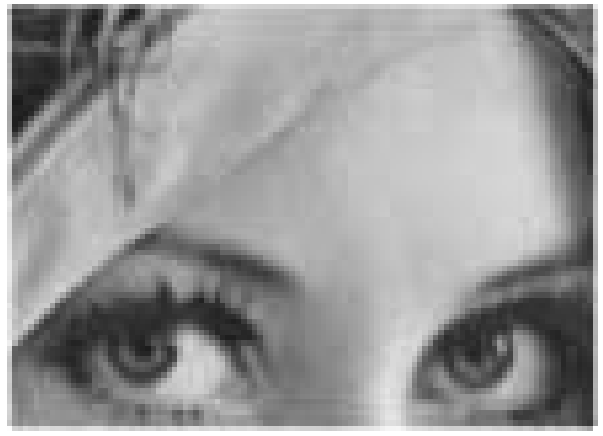

Figure 8: Original Lena image (no distortion)

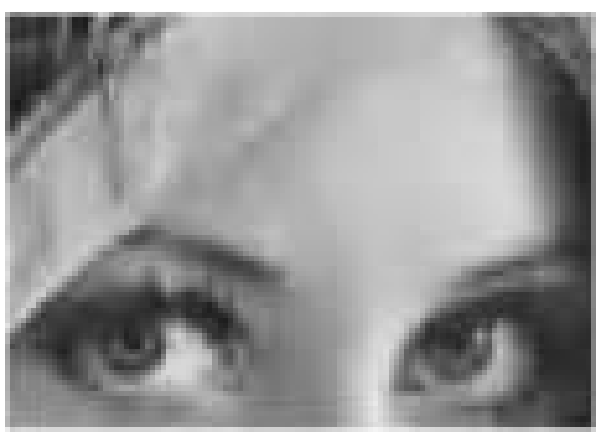

Figure 9: Wavelet compression 1:80 with blurring 
means of improving the received image quality. This can be performed either through image enhancement or image restoration. Image enhancement functions by accentuating specific image features without regard to the technical image fidelity factors, such as peak signal to noise ratio (PSNR.) Image restoration works by recovering a better image from the degraded one by using a model for the degradation process.

An edge construction model was proposed by $\mathrm{Fan}^{14}$ as a post processing technique for image restoration. This model involved analysing the degradation of edges using the wavelet transform and the commonly implemented zerotree coding and quantisation technique. ${ }^{8}$ The edge recovery technique was implemented by estimating the original edge model parameters from the compressed image. This was based on modelling edges as Gaussian smoothed step functions and using a 2-dimensional Gaussian filter to reduce the noise present across the edges. This work was aimed at obtaining a tradeoff between perceived reconstructed image quality and the image Power Signal to Noise Ratio (PSNR.)

\subsection{Reconstruction of Lost Image Blocks - An Extension to Source Coding}

Once data has been compressed into a bitstream it becomes extremely sensitive to errors. A lot of research has been put into channel coding (also referred to as Forward Error Correction, FEC), which is a solution based on adding redundancy into the data stream. This gives the decoder a better chance of detecting and correcting errors if they occur. Despite increasing the bandwidth requirements of the communications channel and increasing the complexity of the system, this strategy effectively allows for a receiving device to detect and correct errors introduced by the channel. ${ }^{15}$ Unfortunately, these techniques cannot completely prevent the loss of image blocks if the Bit Error rate (BER) is unknown, which characterises most wireless scenarios However, as a consequence of the fading that characterises wireless environments. The loss of image blocks typically occurs in a random and bursty fashion, degrading the image quality. Rane et al ${ }^{16}$ showed that the lost image blocks could be reconstructed from available surrounding blocks using a process of interpolation using very simple techniques.

\subsubsection{Classification of the blocks}

The first step to take in reconstructing the lost blocks as proposed by Rane et $a l^{16}$ involved classifying the blocks as either part of an edge or non-edge. By referring to Section 3.1, we see the magnitude of each wavelet coefficient indicates the amount of change, as well as the location at which the change occurs. This can be illustrated in Figure 10, where shaded areas indicate high coefficients as a result of a vertical edge contained in an $8 \times 8$ block. The level 2 coefficients obtained from the first wavelet decomposition (implemented in Figure 7) represent

\begin{tabular}{|c|c|c|c|}
\hline DC & Ho & \multirow{2}{*}{ H1 } & \multirow[t]{3}{*}{ Horizontal Detail } \\
\hline V0 & D0 & & \\
\hline \multicolumn{2}{|c|}{ V1 } & D1 & \\
\hline \multicolumn{3}{|c|}{ Vertical Detail } & Diagonal Detail \\
\hline \multicolumn{3}{|c|}{ V2 } & D2 \\
\hline
\end{tabular}

Figure 10: Wavelet decomposition of block containing a vertical edge.

localised spatial detail and would not be suitable for edge detection. Instead, level 1 coefficients extracted from the second wavelet decomposition ${ }^{16}$ could determine the presence of an edge. Each of these coefficients could then be compared to a variable threshold to determine the presence of an edge. Figure 11 illustrates the 4 possible situations where a vertical edge may be detected in which the values of the level 1 wavelet coefficients 

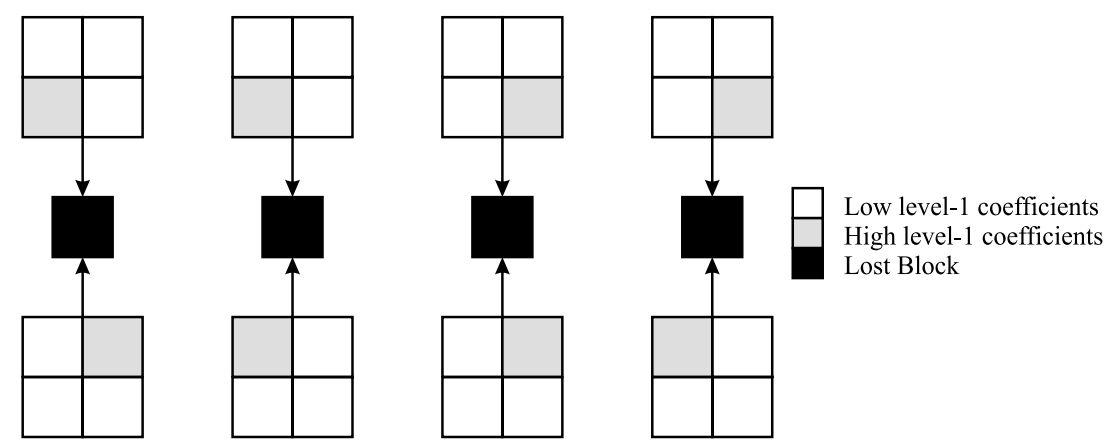

Figure 11: Possible cases for vertical edge detection.

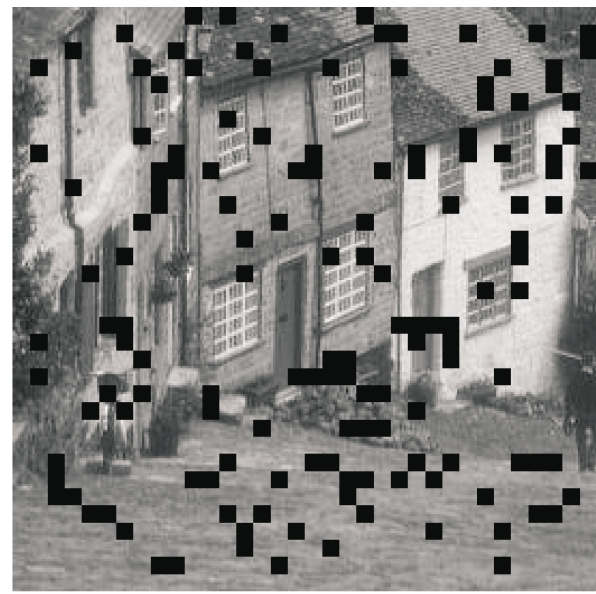

Figure 12: Simulated loss from ${ }^{16}$

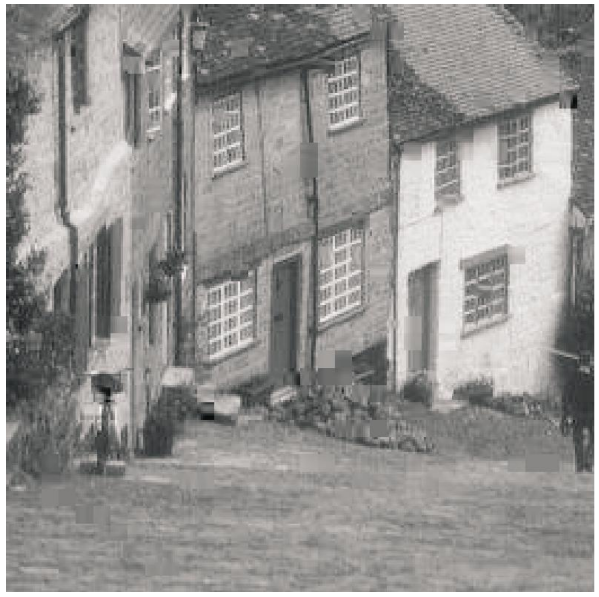

Figure 13: Reconstructed image from ${ }^{16}$

are examined in the closest surrounding $8,8 \times 8$ vertical tiles. This type of edge testing procedure is easily extrapolated for diagonal and horizontal edges.

\subsubsection{Reconstructing blocks containing edges}

The process of reconstructing a lost block containing an edge involved minimising the square of the error of the selected border tiles (shaded in grey in Figure 11 for the four possible vertical edge situations.) This is desirable as it makes the edge through the lost block as consistent as possible with the surrounding edge tiles.

\subsubsection{Reconstruction of non-edge blocks}

Unlike tiles that have been classified as containing edges, non-edge blocks require interpolation from all the surrounding blocks. The D0 level is obtained by averaging the D0 levels of the four diagonal surrounding blocks. The D1 and D2 coefficients are assigned to the closest available diagonal D1 and D2 coefficients. The methods used to interpolate horizontal and vertical details however, are similar to the technique used for edge reconstruction. The results obtained using this type of block reconstruction technique are shown in Figures 12 and 13.

\section{CONCLUSIONS}

The development of wavelet technology for use in images has gained widespread acceptance in signal processing and in image compression research. For the purposes of low bandwidth, wireless systems, wavelet-based image coding schemes such as JPEG2000 outperform other coding schemes. Wavelet basis functions have variable length, as well as position and consequently offer higher obtainable compression levels without objectionable blocking artifacts. Wavelet-based coding also facilitates progressive transmission of images, which makes it 
more practical for any receiving device. Because of the inherent multiresolution nature of wavelets, which is facilitated by the tree-based coding structure, these coding schemes are especially suitable for applications where scalability and tolerable degradation are important. Improvements can still be made to optimise the received quality of wavelet-based image coding for mobile environments. These include innovations to reconstruct fine image detail, such as edges that have been lost as a result of discarding detailed image information during the image encoding process. Block reconstruction is also a very new successful development in conjunction with wavelet-based images. This development has made it possible to approximate image blocks that have been lost due to the fading that characterises wireless environments.

\section{ACKNOWLEDGEMENTS}

This work is gracefully acknowledged by the jointly funding of the Australian Research Council and Motorola Software Corporation. Discussions with Dr Matthew Sorell were of assistance to me during this work.

\section{REFERENCES}

1. C. Christopoulos and A. N. Skodras, "The JPEG2000 still image coding system: An overview," IEEE Transactions on Consumer Electronics 46(4), pp. 1103-1127, 2000.

2. G. Strang, "Wavelets," American Scientist 82, pp. 250-255, 1992.

3. S. Mallatt and W. L. Hwang, "Singularity detection and processing with wavelets," IEEE Transactions on Information Theory 38, pp. 617-643, March 1992.

4. I. Daubechies, Ten Lectures on Wavelets, CBMS-NSF Regional Conference Series in Applied Mathematics, Society for Industrial and Applied Mathematics, 3600 University City Science Center, Philadelphia, Pennsylvania, 1992.

5. K. Ngan, C. Yap, and K. Tan, Video Coding For Wireless Communications Systems, 10, Marcel Dekker Inc, 270 Madison Avenue, New York, NY 10016, 2001.

6. Z. Xiong, K. Ramchandran, M. T. Orchard, and Y.-Q. Zhang, "A comparitive study of DCT and waveletbased image coding," IEEE Transactions on Circuits and Systems for Video Technology 9, pp. 692-695, August 1999.

7. E. Feig, "A fast scaled dct algorithm," in SPIE Image Processing Algorithms and Techniques, 1244, pp. 2-13, Febuary 1990.

8. J. M. Shapiro, "Embedded image coding using zerotrees of wavelet coefficients," IEEE Transactions on Signal Processing 41(12), pp. 3445-3462, 1993.

9. C. D. Creusere, "A new method of robust image compression based on the embedded zerotree wavelet algorithm," IEEE Transactions on Image Processing 6(10), pp. 1436-1442, 1997.

10. C. C. A. N. Skodras, "JPEG2000: The upcoming still image compression standard," in Proceedings of the $11^{\text {th }}$ Portugese Conference on Pattern Recognition, pp. 359-366, May 2000. Porto, Portugel.

11. D. L. Donoho and I. M. Johnstone, "Ideal spatial adaptation via wavelet shrinkage," Biometrika 81, pp. 425455, 1994.

12. S. G. Chang, B. Yu, and M. Vetterli, "Spatially adaptive wavelet threshlding with context modelling for image denoising," IEEE Transactions on Image Processing 9, pp. 1522-1531, September 2000.

13. E. P. Simoncelli, "Modelling the joint statistics of image in the wavelet domain," in Proceedings of the SPIE, 3813, pp. 188-195, 1999.

14. G. Fan and W. K. Cham, "Model-based edge reconstruction for low bit-rate wavelet-compressed images," IEEE Transactions on Circuits and Systems for Video Technology 10(1), pp. 120-125, 2000.

15. A. J. Viterbi, "Convolutional codes and their performance in communication systems," IEEE Transactions on Communications Technology COM-19, pp. 751-772, October 1971.

16. S. D. Rane, J. Remus, and G. Sapiro, "Wavelet-domain reconstruction of lost blocks in wireless image transmission and packet-switched networks," in IEEE International Conference on Image Processing, September 2002, (In Press). 